\title{
Nanoscale Tubular Vessels for Storage of Methane at Ambient Temperatures
}

\author{
Piotr Kowalczyk ${ }^{1 *}$, Lech Solarz ${ }^{2}$, D. D. Do ${ }^{3}$, Adam Samborski ${ }^{1}$, \\ and J. M. D. MacElroy ${ }^{4 *}$
}

(1) Department III, Institute of Physical Chemistry, Polish Academy of Science, Kasprzaka Street 44/52, 01-224 Warsaw, Poland.

(2) Department of Technical Physics, Military Technical Academy, Kaliski Street 2, 00-908 Warsaw, Poland

(3) Department of Chemical Engineering, University of Queensland, St. Lucia, Qld 4072, Australia.

(4) UCD School of Chemical and Bioprocess Engineering, The Centre for Synthesis and Chemical Biology, Conway Institute of Biomolecular and Biomedical Research, University College Dublin, Belfield, Dublin 4, Ireland.

\section{Langmuir}

Number of pages: 18

Number of tables: 1

Number of figures: 7

Number of movies: 2 


\section{SI. Simulation Details}

In the simulation of methane storage in idealized bundles of single-walled carbon nanotubes (SWNTs) and worm-like carbon pores we used a grand canonical ensemble (i.e., fixed system volume $V$, temperature $T$, and the chemical potential of the bulk fluid $\left.\mu_{p}\right)^{1,2}$. We calculated the excess part of the chemical potential of methane in the canonical ensemble according to Widom's particle insertion method and the corresponding bulk pressure of hydrogen from the virial theorem ${ }^{1,3}$. As usual, we added the tail corrections for the energy and pressure after the simulation has been completed ${ }^{1,2}$. To simulate the storage of methane in an infinitely long bundle of SWNTs and worm-like carbon pores we adopted a cubic simulation box of size: $52.68 \times 30.41 \times 36.48 \AA$ with periodic boundary conditions in all directions (see Figure 1S). Moreover, for computation of molecular interactions we used the 'minimum image convention' ${ }^{1}$. In the cubic simulation box we placed an idealized hexagonal bundle of $(10,10)$ armchair-type SWNTs consisting of 11 tubes (see Figure 1S). Following Tanaka et al. ${ }^{4}$, we used a van der Waals gap of $4 \AA$ between the individual $(10,10)$ armchair-type SWNTs of internal radii $6.78 \AA$ A. In computing the fluid-fluid interactions we applied a cutoff distance equal $5 \sigma_{f f}\left(\sigma_{f f} \text { is the collision diameter of methane }\right)^{1,2}$. As in previous studies ${ }^{5}$, we did not add the long-range corrections because the fluid within the pores is inhomogeneous. Both the pressure and chemical potential of methane calculated in the canonical ensemble were used as an input to the GCMC simulations. To trace the isotherms, we mimic experimental procedures by starting with an empty simulation box. Simulation runs were performed at gradually increasing chemical potentials, and the resulting configurations of one condition were used to initiate the subsequent calculations at higher chemical potentials. In the grand canonical ensemble $8 \times 10^{7}$ configurations were generated. We discarded the first $5 \times 10^{7}$ configurations to guarantee equilibration, whereas the latter $3 \times 10^{7}$ configurations were used to obtain the desired thermodynamic properties. For arbitrary selected points we stored the fluctuations in the total energy. Next, we analyzed the variation of the internal energy to ensure that thermodynamic equilibrium was achieved. 


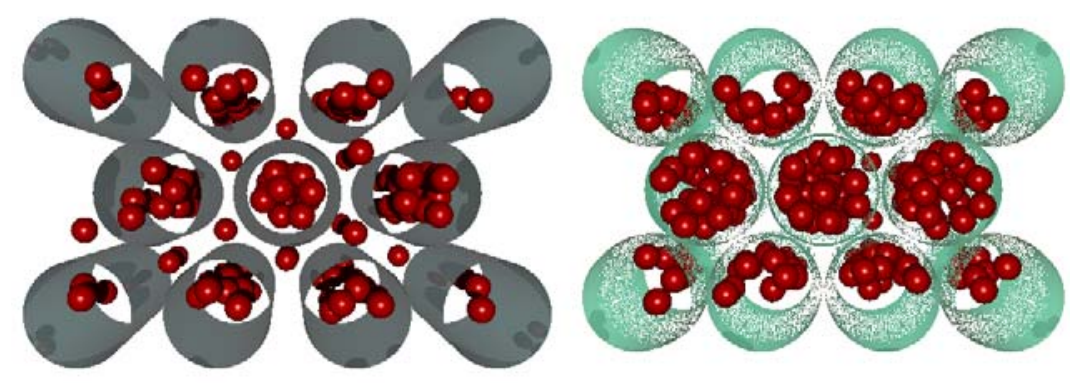

Figure 1S. The model of an idealized bundle of $(10,10)$ SWNTs (left) and worm-like pores (right) used in the current study.

\section{SII. Fluid-Fluid Interaction Potential}

Similarly to Kowalczyk et al. ${ }^{5}$ and Cracknell et al. ${ }^{6}$ we modeled the fluid-fluid interactions via the effective truncated one-centre Lennard-Jones potential (i.e. we treated the methane as a quasi-spherical molecule),

$V_{f f}=4 \varepsilon_{f f}\left[\left(\frac{\sigma_{f f}}{r}\right)^{12}-\left(\frac{\sigma_{f f}}{r}\right)^{6}\right] \Theta\left(r_{\text {cut }}-r\right)$

Here, $r$ is the distance between two interacting fluid molecules, $\sigma_{f f}$ denotes the LennardJones collision diameter, $\varepsilon_{f f}$ is the Lennard-Jones well-depth, $r_{c u t}=5 \sigma_{f f}$ is the cutoff distance, and $\Theta$ denotes Heaviside function. Lennard-Jones parameters $\sigma_{f f}=3.81 \AA$ and $\varepsilon_{f f} / k_{b}=148.12 \mathrm{~K}$ (here, $k_{b}$ denotes the Boltzmann's constant) for methane interactions were taken from the previous study ${ }^{5,6}$.

In the canonical ensemble we simulated the bulk properties of methane at $293 \mathrm{~K}$ up to $80 \mathrm{MPa}$. We used both $(\mathrm{N}, \mathrm{E}, \mathrm{T})$ and $(\mathrm{N}, \mathrm{p}, \mathrm{T})$ Monte Carlo Ensembles. Furthermore, we measured the excess part of the chemical potential by the Widom's particle insertion method ${ }^{3}$. Note that the simple one-centre Lennard-Jones potential describes the experimental data very well and as a result validates the selected model of the fluid-fluid interaction (see Figure $2 \mathrm{~S}$ 
and $3 \mathrm{~S}$ ). As has been recently shown by Do and Do, the one-centre Lennard-Jones model generates similar predictions for the methane uptake in slitlike carbon pores in comparison to the five-centre potential function ${ }^{7}$.

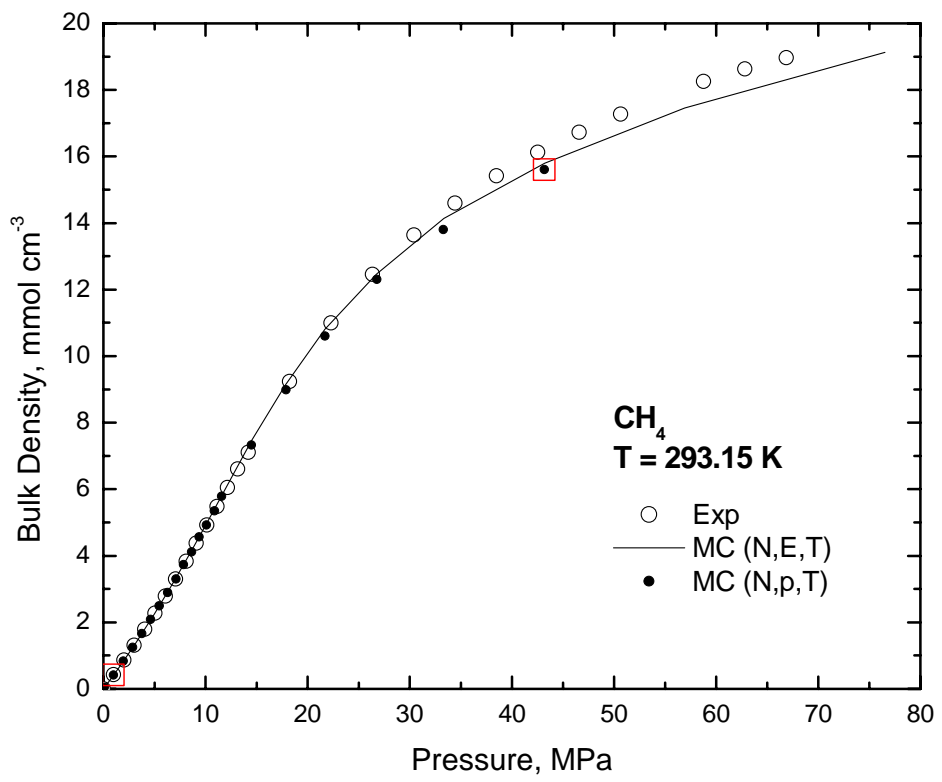

Figure 2S. Comparison of the experimental equation of state for methane at $T=293 \mathrm{~K}$ (open circles) with the results calculated from Monte Carlo simulations in canonical ensemble (solid line $-(\mathrm{N}, \mathrm{E}, \mathrm{T})$ and close circles $-(\mathrm{N}, \mathrm{p}, \mathrm{T}))$.

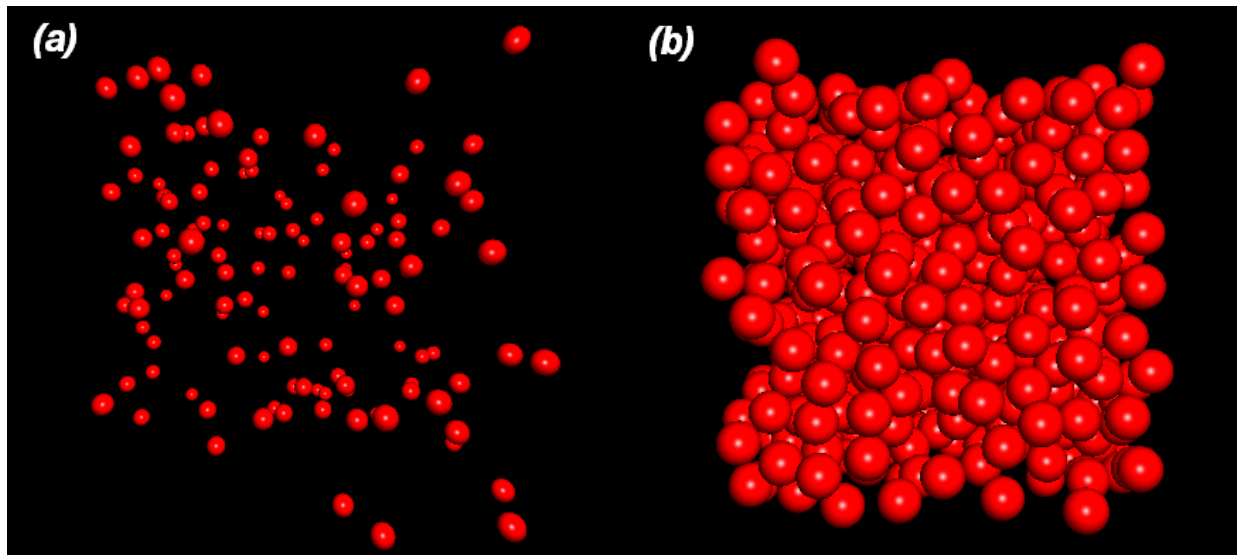

Figure 3S. Snapshot of methane in the bulk phase at (a) - $1 \mathrm{MPa},(\mathrm{b})-43.2 \mathrm{MPa}$ and $293 \mathrm{~K}$ collected from the (N, V, T) Monte Carlo Simulation. The selected points correspond to red triangles from Figure 2S. 


\section{SIII. Solid-Fluid Interaction Potential for Worm-like Pores}

As in the work of Steele and Bojan ${ }^{8}$ we have adopted structureless models for the individual straight or worm-like tubes investigated in these studies. This assumption is realistic for high temperatures due to the high thermal energy of the fluid particles. In other words the oscillations of the solid-fluid potential generated by the graphite are small. Moreover, this approximation is acceptable since the fluid molecule is large relative to the spacing between the surface atoms $\left(d_{l} / \sigma_{f f}=0.37\right.$, where $d_{l}=0.142 \mathrm{~nm}$ denotes the C-C distance in graphite). Following the accepted method for modelling the solid-fluid interactions, we replaced the sum over interactions of a given fluid particle with all of the solid atoms by an integral. In accord with Steele and Bojan ${ }^{8}$ we assumed that the surface density of carbon atoms smeared on the wall of the worm-like carbon tubes is $\rho_{s}=0.382$ atoms $/ \AA^{2}$ (i.e. the same as in graphite). We have defined the position of the fluid molecule inside/outside the infinitely long structureless worm-like pore by the coordinates $\left(x_{w}, y_{w}, z_{w}\right)$, whereas a point on the surface has coordinates $\left(x_{p}, y_{p}, z_{p}\right)$.

(a)

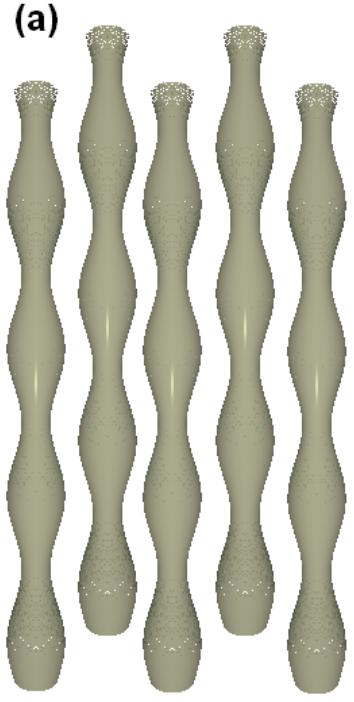

(b)

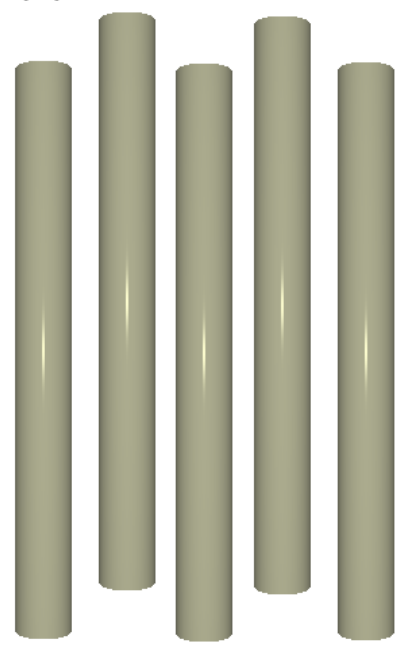

(c)

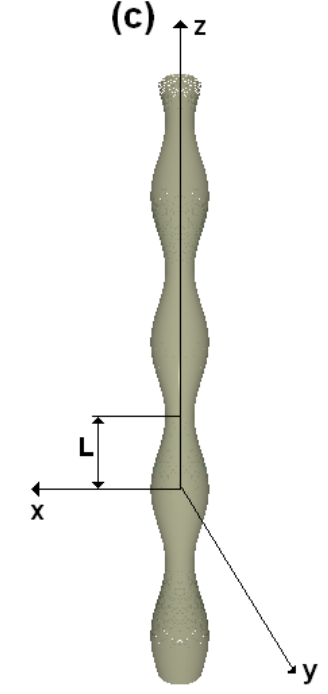

(d)

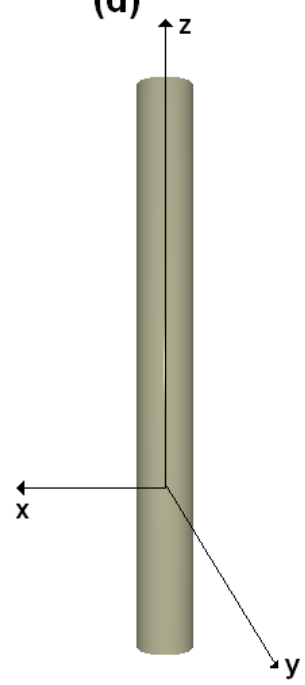

Figure 4S. The model of infinitely long structureless idealized bundles of single-walled worm-like pores [panel (a)], nanotubes [panel (b)], an individual worm-like pore [panel (c)] used for the calculation of the solid-fluid interaction potential, and an individual cylindrical pore [panel (d)] used for the calculation of the solid-fluid interaction potential. 
The infinitely long structureless worm-like pore is characterized by a radius defined as follows,

$$
R(a ; b, z) \equiv R=a+b \sin \left(2 \pi \frac{z}{L}\right)
$$

Here, $a>b$ are parameters, $L$ denotes the length of the basic periodic unit (see Figure 4S panel [c]). Obviously, putting $b=0$ the infinity long structureless worm-like pore is reduced to infinitely long straight structureless cylinder and the derived formula for the total solidfluid interaction potential is equivalent to that derived by Steele and Bojan ${ }^{8}$. For computational purposes we assumed that $z \in\left[D_{\min }, D_{\max }\right]$, where $D_{\min }=z_{w}-M L$ $D_{\max }=z_{w}+M L$ (i.e. we considered an infinitely long structureless pore as a long finite one). We have found that $M=10$ is sufficient for calculation of the total solid-fluid interaction potential in an infinitely long structureless worm-like pore due to the rapid decay in the dispersion interactions.

In cylindrical coordinates the surface integral can be defined as follows,

$$
V_{s f}(R) \equiv \rho_{s} \int_{S} V(r) d S=4 \varepsilon_{s f} \rho_{s} \int_{z_{w}}^{z_{w}+M L} R \sqrt{1+\left(b \frac{2 \pi}{L}\right)^{2} \cos ^{2}\left(2 \pi \frac{z_{p}}{L}\right)} d z_{p}\left\{\sigma_{s f}^{12} \int_{0}^{2 \pi} \frac{d \gamma}{(a+b \cos \gamma)^{6}}-\sigma_{s f}^{4} \int_{0}^{2 \pi} \frac{d \gamma}{(a+b \cos \gamma)^{4}}\right\}
$$

where $\sigma_{s f}$ and $\varepsilon_{s f}$ denotes Lennard-Jones solid-fluid collision diameter and well-depth, respectively.

The internal integrals can be solved analytically,

$$
\int_{0}^{\pi} \frac{d x}{(a+b \cos (x))^{n+1}}=\frac{1}{2} \int_{0}^{2 \pi} \frac{d x}{(a+b \cos (x))^{n+1}}=\frac{\pi}{2^{n}(a+b)^{n} \sqrt{a^{2}-b^{2}}} \sum_{k=0}^{n} \frac{(2 n-2 k-1) ! !(2 k-1) ! !}{(n-k) ! k !} \cdot\left(\frac{a+b}{a-b}\right)^{k}
$$

$$
\int_{0}^{2 \pi} \frac{d x}{(a+b \cos (x))^{6}}=\frac{\pi}{16(a+b)^{5} \sqrt{a^{2}-b^{2}}}\left[\begin{array}{l}
\frac{945}{120}+\frac{105}{24}\left(\frac{a+b}{a-b}\right)+\frac{45}{12}\left(\frac{a+b}{a-b}\right)^{2}+\frac{45}{12}\left(\frac{a+b}{a-b}\right)^{3}+\ldots \\
\ldots+\frac{105}{24}\left(\frac{a+b}{a-b}\right)^{4}+\frac{945}{120}\left(\frac{a+b}{a-b}\right)^{5}
\end{array}\right]
$$




$$
\int_{0}^{2 \pi} \frac{d x}{(a+b \cos (x))^{4}}=\frac{\pi}{4(a+b)^{3} \sqrt{a^{2}-b^{2}}}\left[\frac{15}{6}+\frac{3}{2}\left(\frac{a+b}{a-b}\right)+\frac{3}{2}\left(\frac{a+b}{a-b}\right)^{2}+\frac{15}{6}\left(\frac{a+b}{a-b}\right)^{3}\right]
$$

The equation developed above describes the total solid-fluid interaction potential of a fluid molecule placed inside/outside an infinitely long structureless worm-like pore. The parameters of the solid-fluid potential can be obtained from the Lorentz-Berthelot mixing rules, $a b$ initio calculations based on the quantum mechanics, or experimental measurements. In the current work we used the Lorentz-Berthelot mixing rules.

The volume of the individual worm-like pore can be calculated from the following expression,

$$
V=\pi \int_{\alpha}^{\beta}[a+b \sin (k \cdot z)]^{2} d z
$$

where $k=2 \pi / L$. The integral given by Eq. 7 can be solved analytically,

$$
\int[a+b \sin (k \cdot z)]^{2} d z=\frac{a^{2} \cdot k \cdot z+2 b \cdot a \cdot \cos (k \cdot z)+b^{2} \cdot(-(1 / 2) \cos (k \cdot z) \sin (k \cdot z)+(1 / 2) \cdot k \cdot z)}{k}
$$

The surface area of the individual worm-like tube can be calculated numerically from,

$$
S=2 \pi \int_{\alpha}^{\beta} R d l=2 \pi \int_{\alpha}^{\beta}[a+b \cdot \sin (k \cdot z)] \sqrt{1+(b \cdot k \cos (k \cdot z))^{2}} d z
$$

where $k=2 \pi / L$.

Putting $b=0$ in Eq. 2 leads to the simple formulae for the volume and surface area of the straight cylinder of length $L$,

$V=\pi \cdot R^{2} \cdot L$

$S=2 \pi \cdot R \cdot L$ 
As we mentioned above, Eq. 3 is considered to be applicable to simulation studies of adsorption of classical Lennard-Jones molecules at higher temperatures. Lowering the temperature leads to the increasing importance of surface details (i.e. preferential adsorption sites). Physically, the fluid molecules can be simply trapped on the most energetic centre of the solid surface and cannot escape due to low thermal motion. In such cases, the solid surface should be modeled by individual atoms. The model of the infinitely long structureless tube is frequently used in theoretical studies of fluid adsorption in different kinds of carbon nanotubes and generates reliable results ${ }^{10-15}$.

\section{SIV. Investigated Samples of Carbon Nanomaterials}

At first, we investigated the storage of methane at $293 \mathrm{~K}$ in an idealized bundle of (10, 10) armchair-type SWNTs. Carbon nanotubes are characterized by a so-called chiral vector of the nanotube, $C_{h}$. This vector is defined by $C_{h}=n \vec{a}_{1}+m \vec{a}_{2}$, where $\vec{a}_{1}$ and $\vec{a}_{2}$ are unit vectors in the two-dimensional hexagonal lattice. Here $n$ and $m$ are integers. Another important parameter is the chiral angle, which is the angle between $C_{h}$ and $\vec{a}_{1}$. Armchair nanotubes are formed when $n=m$ and the chiral angle is $30^{\circ}$.From the above definition, (10, 10) armchair-type SWNTs are characterized by $C_{h}=10 \vec{a}_{1}+10 \vec{a}_{2}$. Following on from the cylindrical geometry of a rolled graphite sheet, the radius of $(10,10)$ armchair-type SWNTs is $R_{(10,10)}=6.78 \AA$. Next, we investigated the storage of methane at $293 \mathrm{~K}$ in six samples of an idealized bundle of worm-like carbon pores labeled $0.05,0.1,0.15,0.2,0.25$, and 0.3 . For all samples we kept the same minimal pore radius of individual worm-like carbon pores forming an idealized bundle and progressively increase the maximal pore radius (see Table below),

\begin{tabular}{|c|c|c|c|c|c|c||}
\hline \multirow{2}{*}{ Parameter } & \multicolumn{7}{|c||}{ Label } \\
\cline { 2 - 7 } & 0.05 & 0.1 & 0.15 & 0.2 & 0.25 & 0.3 \\
\hline$R_{\min }, \AA$ & 6.78 & 6.78 & 6.78 & 6.78 & 6.78 & 6.78 \\
\hline$R_{\max }, \AA$ & 7.16 & 7.54 & 7.92 & 8.30 & 8.69 & 9.07 \\
\hline \hline
\end{tabular}


Obviously, there are model parameters that take into account the deformed structure of an infinitely long, idealized bundle of SWNTs. For specified carbon nanomaterials the parameters should be obtained from independent experimental measurements (i.e. scanning electron microscopy, high-resolution transmission microscopy, X-Ray diffraction, etc).

\section{SV. Results - Efficiency of Methane Storage}

The correct evaluation of the total - equilibrium - uptake of methane is a well-known experimental problem. The quantity that is experimentally measurable is the excess of adsorption, which is the amount of adsorbate in excess of that of bulk fluid at the same temperature and pressure, and in the same available volume. Experimentally, the excess of adsorption can only be calculated when the true density of carbon is known. The latter is usually obtained via the helium-displacement method, and the validity of this method has been questioned in the literature. For example, Beenakker et al. ${ }^{16}$ pointed out that the quantum effect for helium placed in small nanopores at room temperature cannot be neglected. Since the thermal de Broglie wavelength for helium at room temperature $\lambda \approx 0.1 \mathrm{~nm}$ approaches the size of the nanopores the adsorbed amount depends on the quantum energy levels of helium. In other words, helium cannot access the smallest pores due to Heisenberg's uncertainty principle, and the helium-displacement method overestimates the density of carbon in the examined material.

Contrary to experimental measurements, GCMC simulation allows for a precise determination of the total uptake of methane i.e. absolute value of adsorption because the geometry of the adsorbent is specified.

The absolute value of adsorption (i.e. total methane uptake) is given by,

$\Gamma_{a b s}=\frac{\langle N\rangle}{V}$

where $\langle N\rangle$ is the ensemble average of the number of methane molecules in the simulation box of volume, $V$. Knowing the absolute value of adsorption the stored volumetric energy can be calculated using the following expression,

$\Omega_{a b s}=\Gamma_{a b s} \cdot \Delta H$ 
Here, $\Delta \mathrm{H}$ denotes the heat of methane combustion (i.e. heat of reaction per mole equates to the difference in enthalpy of the products and the reactants). Following Dell and Rand ${ }^{17}$ the methane heat of combustion is given by,

\begin{tabular}{|c|c|c|c|c|c|c|c|}
\hline \hline & $\mathrm{CH}_{4}$ & + & $2 \mathrm{O}_{2}$ & $\rightarrow$ & $\mathrm{CO}_{2}$ & + & $2 \mathrm{H}_{2} \mathrm{O}$ \\
\hline$\Delta \mathrm{H}_{f}^{0}:$ & -74.85 & & 0 & & -393.5 & & $2 \times-285.83$ \\
\hline
\end{tabular}

Following the above table the total heat of combustion is $-55.6 \mathrm{MJ}$ per $\mathrm{kg}$ methane. Obviously, for the automobile industry the prediction of the storage energy is the most important factor. In fact, the high volumetric energy of methane reduces the size of the tank and extends the distance between refueling. On the other hand, to reduce the mass of the tank the adopted nanoporous material should greatly enhance gravimetric mass of methane per unit mass of adsorbent. GCMC allows us to directly calculate the gravimetric storage, expressed as gravimetric weight percent ${ }^{18}$,

$\rho_{w}=\frac{M_{C_{4}}}{M_{C_{4}}+M_{C}} \cdot 100 w t . \%$

where $M_{\mathrm{CH}_{4}}, M_{\mathrm{C}}$ are the total mass of methane molecules and carbon in simulation box, respectively. Note that gravimetric weight percent is an absolute value of adsorption and not the excess one. The total mass of carbon can be easily computed from the total surface area of carbon in the simulation box (see Figure 1S),

$S_{\text {tot }}=6 \cdot S$

Here, $S$ is given by Eq. 9 and 11 for idealized bundle of worm-like pores and SWNTs, respectively. Knowing $S_{\text {tot }}$ we can calculate the total weight of carbon in the unit cell,

$w_{c}=\frac{\rho_{s}}{N_{a}} S_{c} m_{c}$ 
Here $m_{c}$ denotes molar mass of carbon $\left(m_{c}=12.01 \mathrm{~g} \mathrm{~mol}^{-1}\right)$, and $N_{a}$ is the Avogadro's number.

To estimate the efficiently of the storage of methane fluid by a nanoporous material we have to calculate the Gibbs excess value of adsorption. From GCMC simulation we can calculate the Gibbs excess value of adsorption per unit surface area from the following expression $^{19-21}$,

$\Gamma_{e x c}=\frac{\langle N\rangle-\rho_{b} V}{S_{\text {tot }}}$

where $\rho_{b}$ denotes bulk density of methane and $V$ is the total volume of the simulation box. Summing up, Eqs. 12-17 are necessary for the complete description of the methane storage efficiency by nanoporous materials.

\section{SVI. Results - The Binding Energy of a Nanomaterial}

The binding energy of a nanomaterial, which measures the strength of the methanesolid surface interactions, is an important quantity in the study of fluid storage. For the automobile industry the 'host nanomaterial' should greatly enhance the density of stored fluid (high binding energy) and the physical attraction caused by the van der Waals forces is the most suitable mechanism for controlled loading/release of stored fuel. Moreover, adsorption is an exothermic process and the released heat should be taken into account.

From fluctuation theory, we can calculate the isosteric heat of adsorption form the following expression,

$$
q_{s t}=k_{b} T+\frac{\langle U\rangle\langle N\rangle-\langle U N\rangle}{\left\langle N^{2}\right\rangle-\langle N\rangle\langle N\rangle}
$$

where $\langle\ldots\rangle$ denotes ensemble average, $k_{b}$ is the Boltzmann constant, $N$ is the number of particles, and $U$ is the configuration energy of the simulated system. We can divide the configuration energy into two contributions; one due to fluid-fluid interactions while the other 
is due to the fluid-solid interactions, that is, $U=U_{f f}+U_{s s}$. Finally, Eq. 18 can be rewritten as follows,

$$
q_{s t}=\left[k_{b} T+\frac{\left\langle U_{f f}\right\rangle\langle N\rangle-\left\langle U_{f f} N\right\rangle}{\left\langle N^{2}\right\rangle-\langle N\rangle\langle N\rangle}\right]+\frac{\left\langle U_{s f}\right\rangle\langle N\rangle-\left\langle U_{s f} N\right\rangle}{\left\langle N^{2}\right\rangle-\langle N\rangle\langle N\rangle}
$$

Eq. 19 is very important for the interpretation of the processes accompanying the transfer of fluid molecules from the bulk phase to the solid surface.

The binding energy can be measured calorimetrically and can be used for the validation of the adopted force filed. Unfortunately, calorimetric measurements are much more difficult and time-consuming in contrast to standard entropic ones (i.e. measurement of the adsorption isotherms $)^{22}$. On the other hand, the binding energy can be calculated from a collection of adsorption isotherms measured at different temperatures by the ClausiusClapeyron equation ${ }^{22}$.

\section{SVII. Results - Specific Heat at Constant Volume}

Another experimentally accessible quantity is the specific heat at constant volume. It defines the reaction of the system when temperature is perturbed, so it characterizes the stability of the system as a function of temperature. Obviously, specific heat characterizes the susceptibility of the system to undergo transformations: capillary condensations, melting phase transitions, layering phase transitions. We can calculate the specific heat at constant volume from fluctuations of the configurational energy and the number of molecules $b^{23}$,

$$
C_{V}=\frac{3}{2}\langle N\rangle k_{b}+\frac{1}{k_{b} T^{2}}\left(\begin{array}{l}
\left\langle U_{f f}^{2}\right\rangle-\left\langle U_{f f}\right\rangle^{2}+\left\langle U_{s f}^{2}\right\rangle-\left\langle U_{s f}\right\rangle^{2}+2\left(\left\langle U_{f f} U_{s f}\right\rangle-\left\langle U_{f f}\right\rangle\left\langle U_{s f}\right\rangle\right) \\
-\frac{\left[\left\langle U_{f f} N\right\rangle-\langle N\rangle\left\langle U_{f f}\right\rangle+\left\langle U_{s f} N\right\rangle-\langle N\rangle\left\langle U_{s f}\right\rangle\right]^{2}}{\left\langle N^{2}\right\rangle-\langle N\rangle^{2}}
\end{array}\right)
$$

Under supercritical conditions the specific heat at constant volume should inform us about the packing of the molecules within nanospaces. Due to the confinement effect the freedom of molecule is restricted and the variation of $C_{v}$ in a nanoporous system should be different from that of the corresponding bulk phase. 
SVIII. Results - Additional Figures.

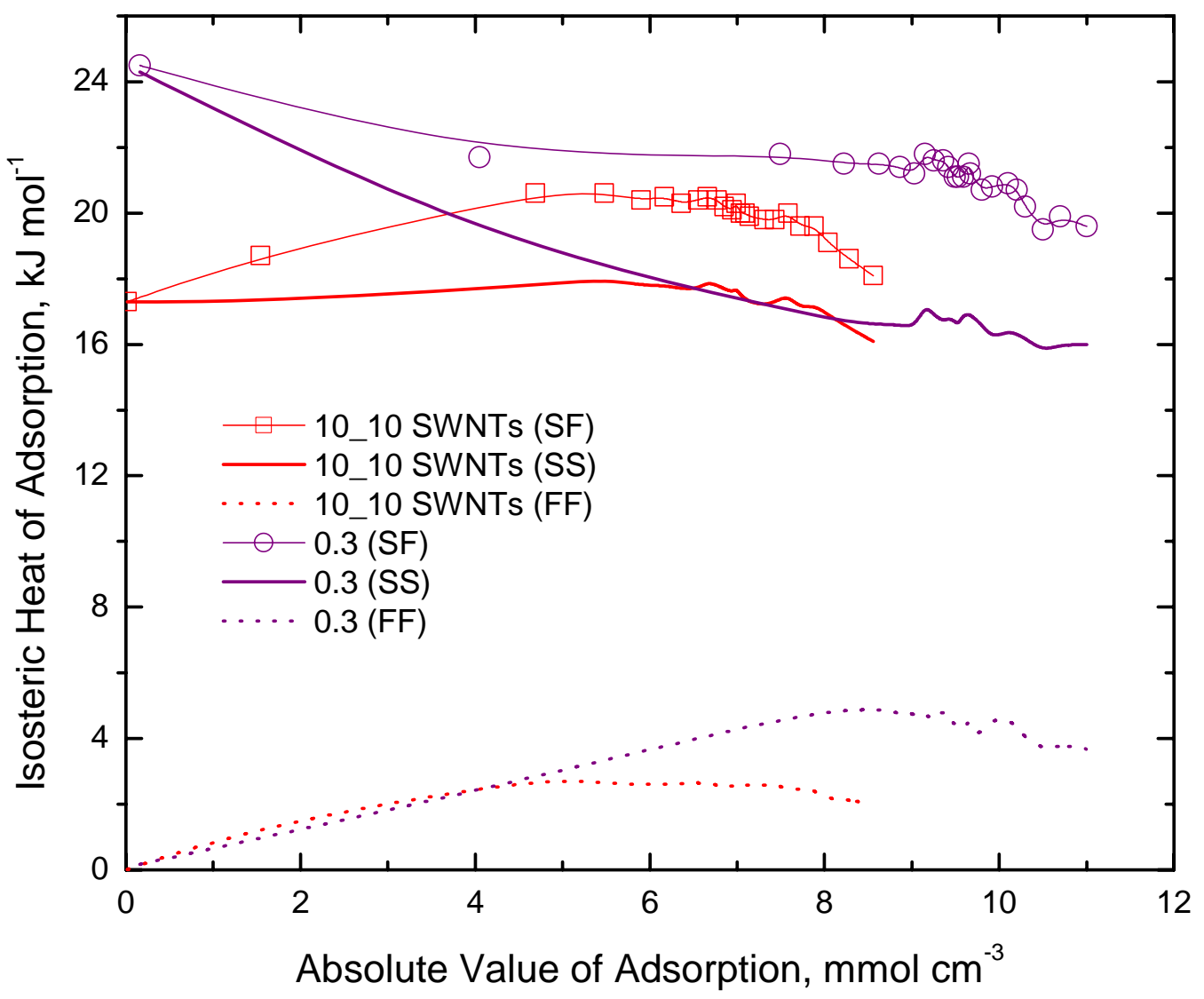

Figure 5S. Isosteric heat of methane versus loading at $293 \mathrm{~K}$ : FF - fluid-fluid contribution, SS - solid-fluid contribution, SF - total isosteric heat of adsorption. 


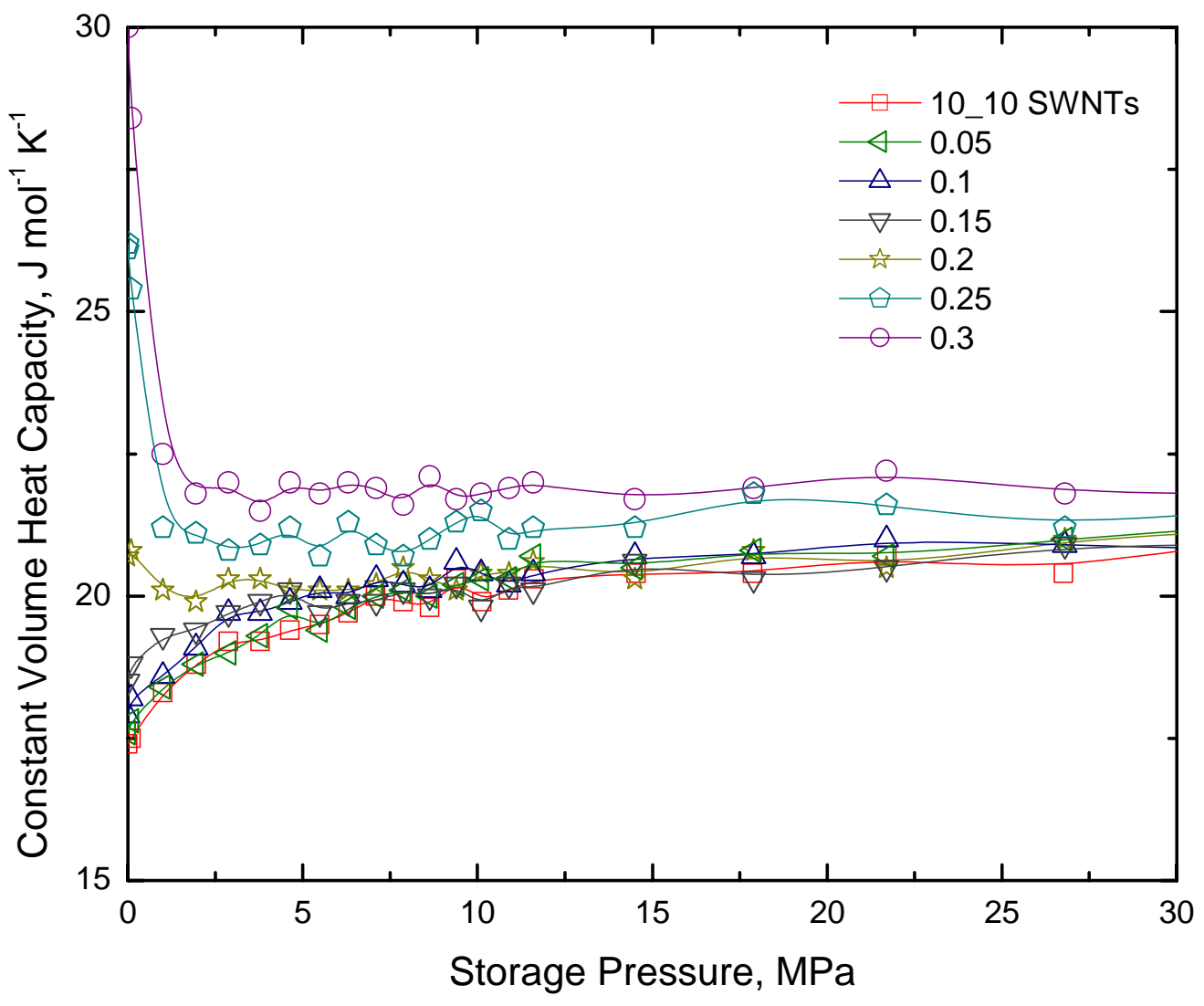

Figure 6S. Constant volume heat capacity versus storage pressure for all investigated samples of carbon nanomaterials. For bulk methane at zero coverage $C_{v}=12.5 \mathrm{~J} \mathrm{~mol}^{-1} \mathrm{~K}^{-1}$. 


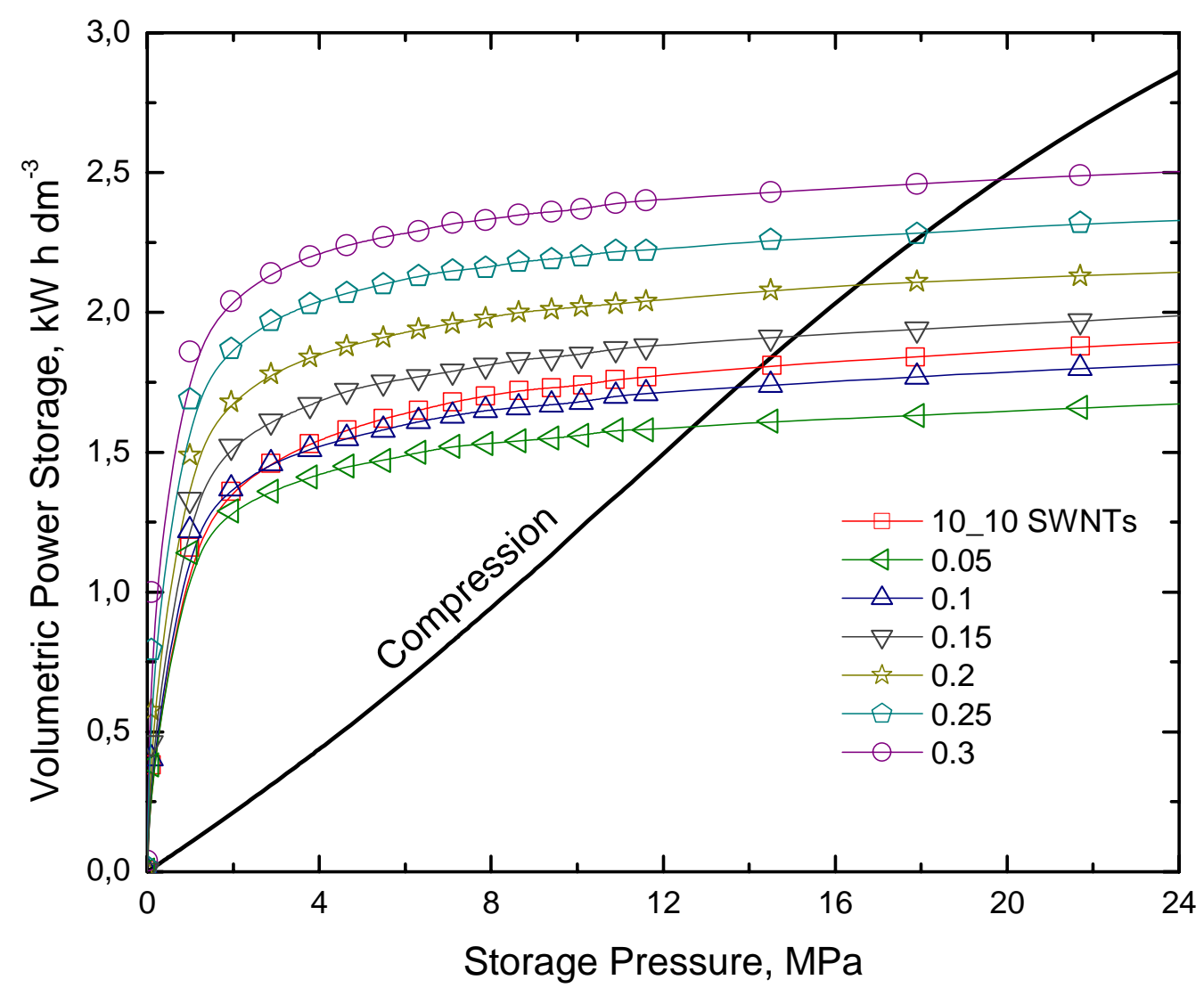

Figure 7S. The stored volumetric power of methane adsorbed in idealized bundles of SWNTs and worm-like pores at $293 \mathrm{~K}$. 


\begin{tabular}{|c|c|c|c|c|c|c|c|c|c|}
\hline \multirow[b]{2}{*}{ Parameter } & \multicolumn{9}{|c|}{ Storage Method } \\
\hline & $\begin{array}{c}\text { Efficiency } \\
\text { of an } \\
\text { engine, } \%\end{array}$ & Compress. & SWNT & 0.05 & 0.1 & 0.15 & 0.2 & 0.25 & 0.3 \\
\hline \multirow{3}{*}{$\begin{array}{c}\text { Volumetric } \\
\text { energy, } \\
\mathrm{MJ} \mathrm{dm}^{-3}\end{array}$} & 100 & 0.37 & 4.18 & 4.09 & 4.38 & 4.80 & 5.37 & 6.07 & 6.68 \\
\hline & 30 & 0.11 & 1.25 & 1.23 & 1.31 & 1.44 & 1.61 & 1.82 & 2.00 \\
\hline & 91.9 & 0.34 & 3.84 & 3.76 & 4.03 & 4.41 & 4.93 & 5.58 & 6.14 \\
\hline \multirow{3}{*}{$\begin{array}{l}\text { Volumetric } \\
\text { power, } \\
\mathrm{kW} \mathrm{h} \mathrm{dm}{ }^{-3}\end{array}$} & 100 & 0.10 & 1.16 & 1.14 & 1.22 & 1.33 & 1.49 & 1.69 & 1.86 \\
\hline & 30 & 0.03 & 0.35 & 0.34 & 0.37 & 0.4 & 0.45 & 0.51 & 0.56 \\
\hline & 91.9 & 0.09 & 1.07 & 1.04 & 1.12 & 1.22 & 1.37 & 1.55 & 1.71 \\
\hline \multirow{3}{*}{ 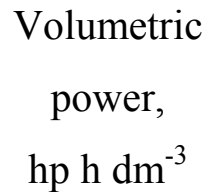 } & 100 & 0.14 & 1.56 & 1.52 & 1.63 & 1.79 & 2.0 & 2.26 & 2.49 \\
\hline & 30 & 0.04 & 0.47 & 0.46 & 0.49 & 0.54 & 0.6 & 0.68 & 0.75 \\
\hline & 91.9 & 0.13 & 1.43 & 1.40 & 1.50 & 1.64 & 1.84 & 2.08 & 2.29 \\
\hline
\end{tabular}

Table 2S. Energy and power storage computed for compressed and adsorbed methane in all of the carbon nanomaterials modeled in this work at $1 \mathrm{MPa}$ and $293 \mathrm{~K}$. Following to Czerwinski $^{24}$, we assumed $30 \%$ efficiency for an internal combustion engine and $91.9 \%$ for a fuel-cell engine. 


\section{References and Notes}

(1) Frenkel, D.; Smith, B. Understanding Molecular Simulation Form Algorithms To Applications; Academic Press: London, 1996.

(2) Allen, M. P.; Tildesley, D. J. Computer Simulation of Liquids; Clarendon: Oxford, 1987.

(3) Widom, B. J. Chem. Phys. 1963, 39, 2808.

(4) Tanaka, H.; Fan, J.; Kanoh, H.; Yudasaka, M.; Iijima, S.; Kaneko, K. Mol. Simul. 2005, $31,465$.

(5) Kowalczyk, P.; Tanaka, H.; Kaneko, K.; Terzyk, A. P.; Do, D. D. Langmuir 2005, 21, 5639.

(6) Cracknell, R. F.; Nicholson, D. Adsorption 1995, 1, 7.

(7) Do, D. D.; Do, H. D. J. Phys. Chem. B 2005, 109, 19288.

(8) Steele, W. A.; Bojan, M. Adv. in Coll. Int. Sci. 1998, 76-77, 153.

(9) Steele, W. A. The interaction of Gases with Solid Surfaces; Pergamon Press, Oxford, 1974.

(10) Ohba, T.; Kanoh, H.; Yudasaka, M.; Iijima, S.; Kaneko, K. J. Phys. Chem. B 2005, 109, 8659.

(11) Kowalczyk, P.; Holyst, R.; Tanaka, H.; Kaneko, K. J. Phys. Chem. B 2005, 109, 14659.

(12) Tanaka, H.; El-Merraoui, M.; Kodaira, T.; Kaneko, K. Chem. Phys. Lett. 2002, 351, 417.

(13) Tanaka, H.; El-Merraoui, M.; Kanoh, H.; Steele, W.A.; Yudasaka, M.; Iijima, S.; Kaneko, K. J. Phys. Chem. B 2004, 108, 17457.

(14) Tanaka, H.; Kanoh, H.; Yudasaka, M.; Iijima, S.; Kaneko, K. J. Am. Chem. Soc. 2005, 127, 7511 .

(15) Tanaka, H.; Murata, K.; Miyawaki, J.; Kaneko, K.; Kokai, F.; Takahashi, K.; Kasuya, D.; Yudasaka, M.; Iijima, S. Mol. Cyst. Liq. Cyst. 2002, 388, 429.

(16) Beenakker, J.J.M.; Borman V.D., Krylov, S.Yu. Chem. Phys. Lett. 1995, 232, 379.

(17) Dell, R.M.; Rand, D.A.J Clean Energy; The Royal Society of Chemistry, Cambridge, 2004.

(18) Guay, P.; Stansfield, B.L.; Rochefort, A. Carbon 2004, 42, 2187.

(19) Kowalczyk, P.; Tanaka, H.; Hołyst, R.; Kaneko, K.; Ohmori, T.; Miyamoto, J. J. Phys Chem. B 2005, 109, 17174.

(20) Levesque, D.; Gicquel, A.; Darkrim, F. L.; Kayiran, S. B. J. Phys. Con. Matt. 2002, 14, 
9285.

(21) Do, D. D.; Do, H. D. Langmuir 2004, 20, 10889.

(22) Gregg, S. J.; Sing, K. W. S. Adsorption, Surface Area and porosity; Academic Press, London, 1982.

(23) Kuchta, B.; Firlej, L.; Maurin, G. J. Chem. Phys. 2005, 123, 174711.

(24) Czerwinski, A. Accumulators, batteries, and cells; WKL: Warsaw, 2005 (in Polish). 